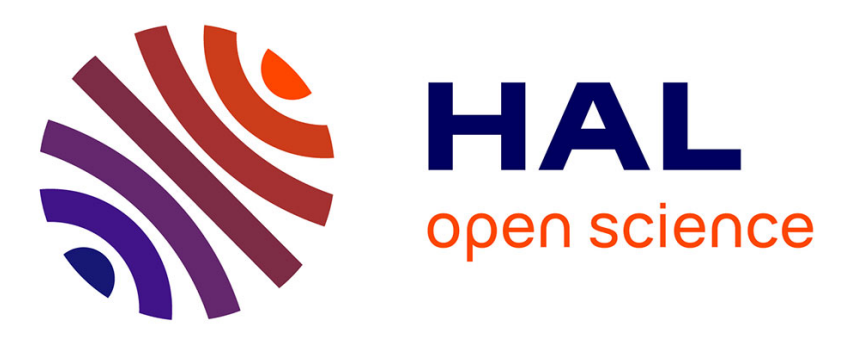

\title{
Reduced Complexity MUD-MLSE Receiver for Partially-Overlapping WLAN-Like Interference
}

Philippe Mary, Jean-Marie Gorce, Guillaume Villemaud, Mischa Dohler, Marylin Arndt

\section{- To cite this version:}

Philippe Mary, Jean-Marie Gorce, Guillaume Villemaud, Mischa Dohler, Marylin Arndt. Reduced Complexity MUD-MLSE Receiver for Partially-Overlapping WLAN-Like Interference. IEEE VTC Spring 2007, Apr 2007, Dublin, Ireland. inria-00399472

\section{HAL Id: inria-00399472 \\ https://hal.inria.fr/inria-00399472}

Submitted on 26 Jun 2009

HAL is a multi-disciplinary open access archive for the deposit and dissemination of scientific research documents, whether they are published or not. The documents may come from teaching and research institutions in France or abroad, or from public or private research centers.
L'archive ouverte pluridisciplinaire HAL, est destinée au dépôt et à la diffusion de documents scientifiques de niveau recherche, publiés ou non, émanant des établissements d'enseignement et de recherche français ou étrangers, des laboratoires publics ou privés. 


\title{
Reduced Complexity MUD-MLSE Receiver for Partially-Overlapping WLAN-Like Interference
}

\author{
P. Mary ${ }^{1,2}$, J.M. Gorce ${ }^{2}$, G. Villemaud ${ }^{2}$, M. Dohler ${ }^{1}$, M. Arndt ${ }^{1}$ \\ ${ }^{1}$ France Télécom R\&D, 28 Chemin du Vieux Chêne, 38243 Meylan Cedex, France \\ ${ }^{2}$ CITI, INSA-Lyon, bat. Léonard de Vinci, 21 Avenue Jean Capelle, 69621 Villeurbanne Cedex, France \\ E-mail: philippe1.mary@ orange-ftgroup.com
}

\begin{abstract}
The roll-out density of wireless local area networks (WLANs) has recently witnessed a dramatic increase and is currently reaching saturation levels. The frequency bands designated to WLANs do thus not suffice anymore to provide nonoverlapping, and hence interference-free, communication bands. A large body of research has been dedicated to a wide variety of optimum maximum likelihood sequence estimation (MLSE) and sub-optimum in-band interference mitigation techniques. Our contribution lies in a reduction of the state-space of a MLSE detector in the case of a desired WLAN receiver experiencing delayed interference from some other transmitters operating in partially overlapping spectral bands and over independent frequency-selecting block-fading channels. Based on the formulation of the optimum receiver, we derive a sub-optimum receiver of reduced complexity and demonstrate its satisfactory performance in the context of strong interference.
\end{abstract}

Index Terms-Interference Cancellation, Wireless Local Area Networks, Multi-User Detector, MLSE, Partial Spectral Overlap

\section{INTRODUCTION}

Wireless local area networks (WLANs), operating at around $2.4 \mathrm{GHz}$, have been widely deployed during the last decade. For instance, France Telecom alone has deployed more than two Million Livebox WLAN systems for domestic and industrial use in the last decade in France.

Victim of its success, the IEEE 802.11 WLAN standard suffers now a lack of radio resources. Although internationally up to 14 mutually overlapping $22 \mathrm{MHz}$ channels are available for IEEE $802.11 \mathrm{~b}$ WLANs occupying a total bandwidth of up to $83.5 \mathrm{MHz}$ at around $2.4 \mathrm{GHz}$, roll-out Engineers nowadays confine themselves to using a few channels only, e.g. the channels numbered 1, 5, 9 and 13 in Europe, so as to limit interference between adjacent cells.

Since four non-overlapping channels clearly do not suffice for the envisaged WLAN deployment scenarios, other means have to be sought to mitigate the limited WLAN system capacity. In this context, two cases of interference can clearly be distinguished [1]: First, if an aggressive roll-out pattern of four with strong co-channel interference (CCI) is being deployed, then (second tier) interfering frequency bands fully overlap with the frequency band of interest. Second, if a less stringent roll-out pattern is being pertained, then (already first tier) interfering frequency bands partially overlap with the band of interest. This late case is referred to as partial channel interference $(\mathrm{PCI})$.
The importance of interference mitigation has been well recognized by the research community and a plethora of milestone contributions has emerged in recent years. Most notably, Sergio Verdú pioneered interference cancelation (IC) methods based on maximum likelihood multi-user detection (MUD) techniques for CDMA-based systems in the early 80s [2], which exhibited prohibitive complexity. Thereupon, a large body of work concentrated on reducing this complexity [3]-[5] and most notably [6], [7]. Resultant and alternative techniques have then mainly been applied to interference mitigation in the context of cellular systems but also WLANs, some important of which are briefly touched upon below.

In many cases, CCI is considered as the main cause of interference [10]-[13]. In [7], the authors suggest to reduce the memory of the CDMA channel in an iterative multiuser detector. However, there are only a few interfering scenarios considered and the reduced state method is not clearly indicated. Although most of the works are related to cellular applications, a linear minimum mean square error (LMMSE) detector is proposed in [9] to deal with the time-dispersive channel and CCI occurring in the ISM band. The proposed detector has been shown to outperform more traditional detectors, e.g. a Rake receiver.

CCI rejection is also accomplished by means of blind and semi-blind IC methods [10], [11], when little or no a priori information is available on individual interferers. Multiple Antenna Interference Cancelation (MAIC) techniques have been introduced to avoid/mitigate strong CCI from a given spatial direction [12]-[14]. Such an approach, however, has its limitations in highly cluttered indoor environments where interference generated by one source often arrives from multiple directions. Furthermore, space-time MUD methods are investigated for the next generation MIMO OFDM-based WLAN systems, i.e. IEEE 802.11n; see, e.g., [15] and [16].

In comparison, less works have been devoted to the case of ACI or PCI mitigation. As already mentioned, PCI plays a crucial role in the limited capacity of WLAN networks. PCI cancelation would allow to increase the reuse factor, thus increasing the global throughput. A maximum-likelihood sequence estimation (MLSE) based on the Ungerboeck formulation has been extended for ACI mitigation in [17] for GSM-type systems. They show that, even for high levels of ACI, significant performance improvements can be achieved with a highly complex MLSE detector, as well as a successive interference canceler of reduced complexity. 
Following the development proposed in [17], this paper assesses the efficiency of PCI mitigation in strongly overlapping channels over severely frequency-selective multipath channels. Due to the differing channel impulse responses (CIRs) for each received signal, we focus on the general case of interfering signals having an asymmetrical cross-correlation (ACC) function.

The paper is structured as follows. The system model with an appropriate MLSE of [17] is described in Section II, where we have augmented the branch metrics to facilitate causal implementation. In Section III, we introduce and discuss the channel synchronization providing a MLSE of reduced complexity. In Section IV, we present and discuss simulation results for our MUD-MLSE applied to WLAN-like systems. Finally, in Section V, conclusions are drawn.

\section{STANDARD MUD-MLSE RECEPTION}

For the sake of completeness, we outline in this section the optimal synchronous MUD-MLSE receiver as partially derived in [2], [17]. We commence with the system model, followed by the augmented derivation of the MUD-MLSE algorithm, which facilitates causal implementation.

\section{A. System model}

We will deal with binary phase shift keying (BPSK) modulation schemes only; an extension to higher order modulations as well as differential formulations is achieved using a similar approach. The BPSK symbol sequence of the $k$-th user, denoted by $\left\{d_{k}(n), n=1, \ldots, N\right\}$ with $N$ being the sequencelength, is shaped by a root raised cosine (RRC) filter with given roll-off factor and up-converted to the $k$-th frequency band. In time-continuous formulation, the transmitted signal is hence expressed as:

$$
x_{k}(t)=\sum_{n} d_{k}(n) h_{f}\left(t-n T_{s}\right),
$$

where $T_{s}$ is the symbol duration and $h_{f}(t)$ is the time-response of the RRC filter.

We assume that there is more than one operational transmitter, where the bands of user $k$ and $l$ are separated by a spectral distance $\Delta f_{k l}$. Note that if there is an equal bandspacing, then $\Delta f_{k l}=\Delta f \cdot(l-k)$, where $\Delta f$ is the minimum band-spacing.

The signal sequence of the active user propagates through a multipath propagation channel with channel impulse response $h_{k}(t)$. At the receiver, additive white Gaussian noise (AWGN), $n(t)$, with a one-sided power spectral density $N_{0}$ is added to the received signal, after which it is amplified, sampled, per user RRC matched filtered, and processed. Assuming user $i$ to be the baseband user of interest, the total baseband signal can be expressed as:

$$
\begin{aligned}
y(t)= & \sum_{n} d_{i}(n) g_{i}\left(t-n T_{s}\right)+ \\
& \sum_{\substack{k \\
k \neq i}} \sum_{n} e^{j 2 \pi \Delta f_{i k} t} d_{k}(n) g_{k}\left(t-n T_{s}\right)+n(t),
\end{aligned}
$$

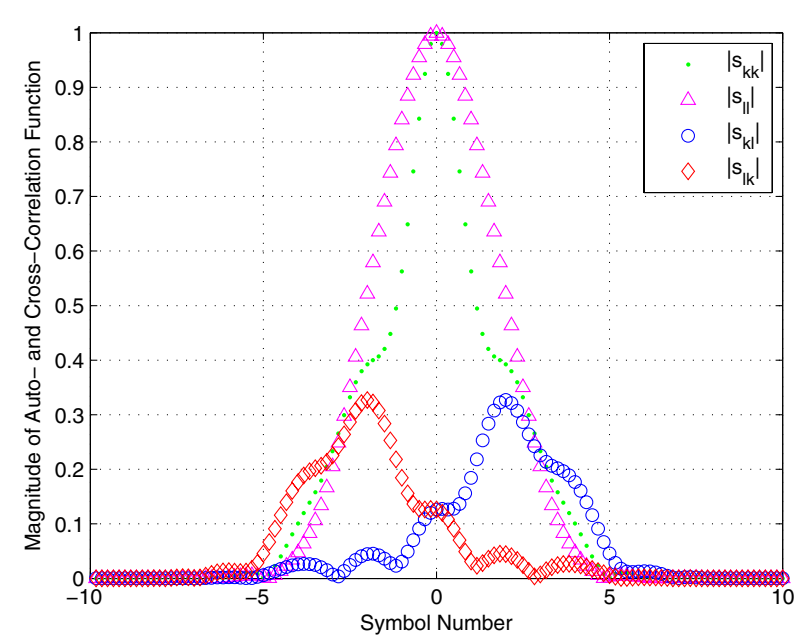

Fig. 1. Generalized auto- and cross-correlation terms of bands $k$ and $l$ with instantaneous channel realizations given in eq. (8).

where $g_{k}(t)=h_{f}(t) \otimes h_{k}(t)$ and $\otimes$ represents the convolution operator. The optimum detection of the desired user in the presence of PCI is achieved by means of a MLSE.

\section{B. MUD-MLSE and Viterbi Branch-Metric}

Maximizing the log-likelihood function of the received data is achieved by maximizing a sum of branch metrics [17]:

$$
J_{H}=\sum_{k} \sum_{n} M_{k}(n)
$$

where $M_{k}(n)$ is given in (4) on top of the next page. In there, $\operatorname{Re}\{\cdot\}$ denotes the real part and * the complex conjugate. Furthermore,

$$
\psi_{k}(n)=\int y(t) e^{-j 2 \pi \Delta f_{i k} t} g_{k}^{*}\left(t-n T_{s}\right) d t,
$$

and the generalised correlation term [17] is given as:

$$
s_{k l}(p)=\left.\int g_{l}(v) e^{j 2 \pi \Delta f_{k l} v} g_{k}^{*}(v-t) d v\right|_{t=p T_{s}} .
$$

The cross-sequence interference is given by the last term in (4). Note, however, that the metric formulation (4) cannot be used directly in a Viterbi algorithm, because the cross-sequence terms are not causal; indeed, the summation with respect to $p$ is done for all $p$. The causal formulation may be easily obtained by moving the non-causal terms in $M_{k}(n)$, involving $d_{l}(n-p)$ with $p<0$, into $M_{l}(n-p)$ leading to a modified branch metric given in (7) on top of the next page and referred to as $M_{k}^{c}(n)$. This detail is provided because it will be used in the next section for ACC interference rejection.

\section{REDUCED STATE-SPACE MUD-MLSE RECEPTION}

\section{A. Viterbi metric derivation for reduced complexity}

The above derived MUD-MLSE is optimum in the minimum error probability sense and hence may exhibit a very large complexity in frequency-selective fading channels requiring a high number of states in the Viterbi decoder. Since the 


$$
M_{k}(n)=\operatorname{Re}\left\{d_{k}^{*}(n) \cdot\left[2 \psi_{k}(n)-d_{k}(n) s_{k k}(0)-2 \sum_{p>0} d_{k}(n-p) s_{k k}(p)-\sum_{\substack{l \\ l \neq k}} \sum_{p} d_{l}(n-p) \cdot e^{j 2 \pi \Delta f_{k l}(n-p) T_{s}} s_{k l}(p)\right]\right\}
$$

$$
M_{k}^{c}(n)=\operatorname{Re}\left\{d_{k}^{*}(n) \cdot\left[2 \psi_{k}(n)-\sum_{l} d_{l}(n) \cdot e^{j 2 \pi \Delta f_{k l} n T_{s}} s_{k l}(0)-2 \sum_{l} \sum_{p>0} d_{l}(n-p) \cdot e^{j 2 \pi \Delta f_{k l}(n-p) T_{s}} s_{k l}(p)\right]\right\}
$$

signal of interest and the interfering signals do not undergo the same wideband fading channel, the instantaneous realizations of the CIR may introduce a significant asymmetry in the crosscorrelation function $s_{k l}(p)$.

As an illustrative example, let us take the instantaneous amplitudes of two symbol-spaced channels:

$$
\begin{aligned}
\left|h_{k}\right| & =\left[\begin{array}{lllll}
0.815 & 0.407 & 0.320 & 0.227 & 0.127
\end{array}\right] \\
\left|h_{l}\right| & =\left[\begin{array}{lllll}
0.227 & 0.460 & 0.688 & 0.460 & 0.227
\end{array}\right]
\end{aligned}
$$

Clearly, in this example, the maximum power taps are not positioned at the same time. Consequently, the $n$-th symbol of the partially overlapping band is not the main interfering symbol at time $n$. In Figure 1, the absolute values of the generalized correlation terms $s_{k k}, s_{l l}, s_{k l}$ and $s_{l k}$ given in (6) are depicted. The maximum energy of the cross-correlation functions are shown to be shifted in opposite directions.

The joint MLSE detector based on (7) takes into account all non zero coefficients of auto and cross correlation function from the $0-$ th delay. This MLSE receiver exhibits a high complexity, the associated state-space increasing exponentially with the time-lag. To decrease the complexity, we propose to limit the state-space of the decoder whilst optimizing the selection of the strongest cross-correlation terms.

For instance, with reference to Figure 1, a full-complexity MUD-MLSE detector ought to have a state-space capturing all auto-interfering paths and also all cross-interfering paths, i.e. $L=5$. A sub-optimal MUD-MLSE would aim at taking into account only the most significant terms. Thus, $L=4$ appears to be a good trade-off if only auto-interference is considered, but fails to remove significant cross-interference.

We hence propose to modify the branch metric (7) so as to synchronize the estimated symbols of each signal in a Viterbi implementation such that the maximum of the intercorrelation terms shifts towards zero. This can be achieved starting from (4) by considering symbol $n+\nu_{k}$ of interfering channel $k$ simultaneously with symbol $n$ in the user channel $i$ with $\nu_{k} \in Z$ in the metric decision. The branch metric is obtained under a causality constraint with respect to this new synchronization, leading to the new branch metric given by (9). This formulation facilitates performance to be traded against complexity.

\section{B. Finding the optimal shift}

In a suboptimal situation, i.e not all cross-correlation coefficients are taken into account, the estimation error is minimised if the shift $\nu_{k}$ is chosen such as to retain the maximum energy of cross-correlation terms. The optimal shift is found by maximizing the energy of cross-correlation terms according to:

$$
\tilde{\nu}_{k}=\underset{\nu_{k} \in Z}{\arg \max } \sum_{p=-(L-1)}^{(L-1)}\left|s_{i k}\left(p+\nu_{k}\right)\right|,
$$

with $\nu_{k} \in Z$ being the considered shift.

Related to this, in [6], the authors suggest an algorithm to reduce the number of states of the trellis diagram based on the knowledge of the CIR at the receiver. The algorithm quantifies the differences between the probability density functions of the correct and incorrect branch metrics in the trellis and allows to reduce significantly the computational complexity. However, this approach is largely more complicated than the herein proposed one, which is simply based on the energy of the CIRs. Furthermore, in [7], the authors suggest to reduce the memory of the CDMA channel in an iterative multiuser detector. The current paper differs from [7], because they reduce the number of states of the Viterbi without considering interference.

\section{Performance Results \& Discussion}

For all simulations, we have used two different power delay profiles. The first channel is a 5-tap symbol-spaced CIR with average powers $[0,-2,-5,-10,-15] \mathrm{dB}$ and simulates a line of sight (LOS) communication between the mobile station (MS) and the access point (AP). A second communication is started between another MS and the same AP on the next channel number. From the receiver point of view, this communication is characterized by a non line of sight (NLOS) propagation with the power delay profile [-10 - $50-2-4] \mathrm{dB}$. Both are blockfading channels, having a total average power normalized to unity. The RRC roll-off factor is fixed at $\alpha=0.33$ and the packet size to 1000 symbols. The symbol duration is assumed to be $90 \mathrm{~ns}$ as a reference to the chip duration in WLAN.

Figure 2 shows the average bit error rate (BER) versus $E b / N o$ obtained with different state-spaces, labelled $L$, and with/without the optimal shift. In this simulation, the signal to interference ratio (SIR) is fixed to $0 \mathrm{~dB}$ and the spectral overlap to about $65 \%\left(\Delta f \cdot T_{s} /(1+\alpha)=0.35\right)$. In all graphs, the curve related to "MLSE with PCI", is the performance of a receiver which does not take into account the interfering adjacent channel but only its own inter-symbol interference (ISI). This curve shows that without interference rejection, the system cannot work. The adaptive shift allows to improve the results for any sub-optimal value of $L(L<5)$. For instance, 


$$
\begin{aligned}
M_{k}^{c}(n)=\operatorname{Re}\left\{d_{k}^{*}\left(n+\nu_{k}\right) \cdot\right. & {\left[2 \psi_{k}\left(n+\nu_{k}\right)-\sum_{l} d_{l}\left(n+\nu_{l}\right) \cdot e^{j 2 \pi \Delta f_{k l}\left(n+\nu_{l}\right) T_{s}} s_{k l}\left(\nu_{k}-\nu_{l}\right)-\right.} \\
& \left.\left.2 \sum_{l} \sum_{\substack{p \\
p>0}} d_{l}\left(n+\nu_{l}-p\right) \cdot e^{j 2 \pi \Delta f_{k l}\left(n+\nu_{l}-p\right) T_{s}} s_{k l}\left(p+\nu_{k}-\nu_{l}\right)\right]\right\}
\end{aligned}
$$

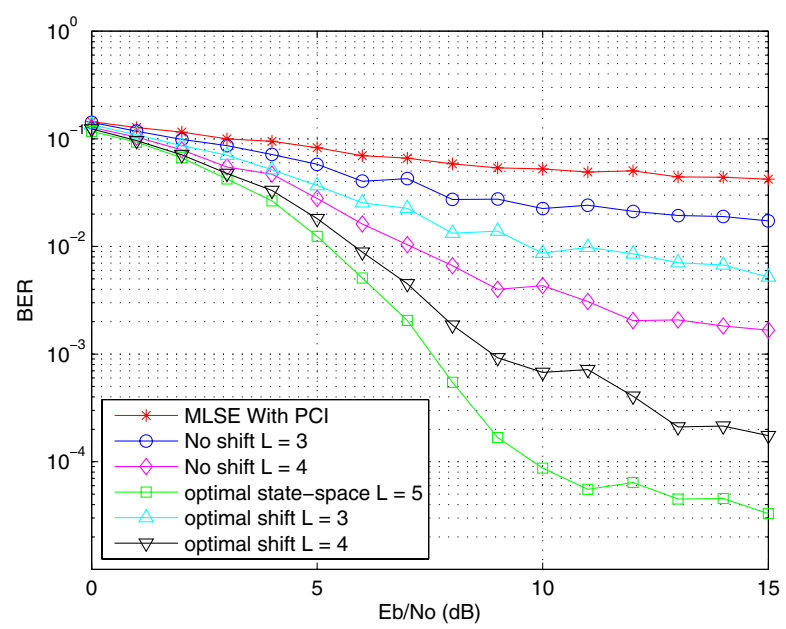

Fig. 2. Average BER versus $E b / N o$, labelled on Viterbi state-space $L$, with and without complexity reduction; channel overlap $=65 \%$, interference power = signal power.

for $E b / N_{o}=15 \mathrm{~dB}$ and $L=4$, the probability of error is reduced by a factor of 10 thanks to the shift.

Figure 3 shows the cumulative density function (CDF) of the BER at $E b / N o=15 \mathrm{~dB}$. Again, for each $L$, the performance with and without optimal shift is provided. The CDF is of interest in the case of block-fading channels, as often occurring in WLAN settings. In such channels, we are not only interested in the average data throughput, but also in the likelihood of having a given error rate at a given $E b / N o$. For the reduced state-space of $L=3$, i.e. only 16 Viterbi states, yields a sufficient outage probability at low BERs. Indeed, in this case, the probability to yield a BER of $10^{-3}$ is $68 \%$ with the optimal shift while only $40 \%$ for the standard approach with $L=3$. If we consider $L=4$, the probability to have a BER less than $10^{-3}$ is more than $95 \%$ with the shifted metric and again less than $85 \%$ with the standard one. When the Viterbi state-space $(L)$ is sufficient, the performance is optimum in the error rate sense.

Figure 4 shows the average BER versus the carrier spacing. The SIR equals $0 \mathrm{~dB}$ and $E b / N o=15 \mathrm{~dB}$. Here, increasing the spectral overlap degrades the performance. But with a carrier spacing of $3 \mathrm{MHz}$ only ( $80 \%$ channel overlap since the bandwidth is approximatively $15 \mathrm{MHz}$ ) a large gain is achieved. Indeed, for $L=4$ the BER obtained with the metric in (9) is by an order of magnitude less: $5 \cdot 10^{-4}$ against $4 \cdot 10^{-3}$ without metric adjustment. We also observe that the BER of a suboptimal Viterbi state-space with and without metric adjustment, i.e. $L=3$ and $L=4$, converge when the

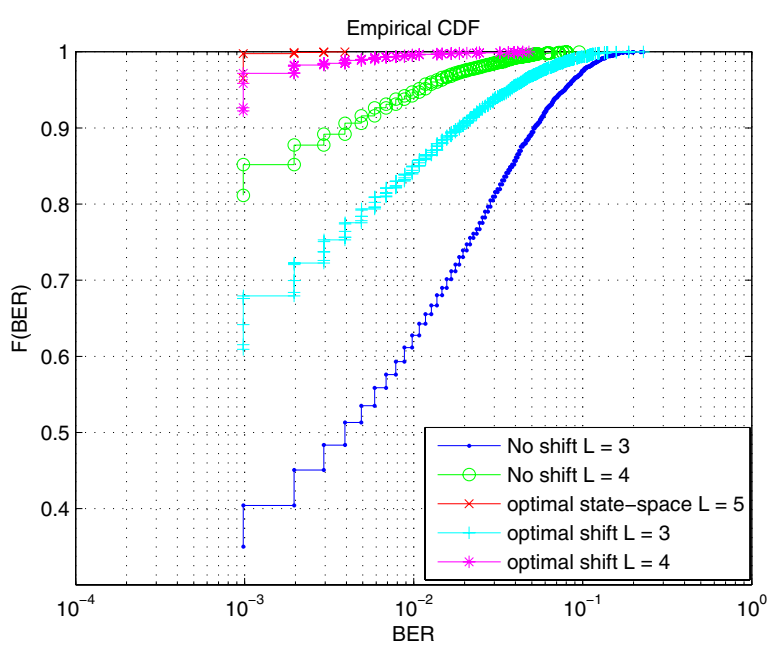

Fig. 3. $\mathrm{CDF}$ of BER at $E b / N o=15 \mathrm{~dB}$, labelled on Viterbi state-space $L$, with and without complexity reduction; channel overlap $=65 \%$, interference power $=$ signal power.

spacing between bands increases. This is due to less interfering signal when the channel overlap decreases, so both metrics are equivalent. We also include the single-user MLSE performance curve. This curve converges towards the performance curve of the MUD-MLSE receiver with $L=5$ without BER floor. The performance enhancement of a receiver based on (9) is pointed out in strong interference conditions (the left part of the graph).

Finally, Fig. 5 shows the average bit error rate versus the average interfering power, with a spectral overlap of $65 \%$ and $E b / N o=15 \mathrm{~dB}$. We observe a performance enhancement for $L=4$ with the shifted metric. A BER reduction of about 10 is achieved when the interfering signal is $5 \mathrm{~dB}$ weaker than the desired signal. When the SIR increases, the augmented metric and the classical one converge. The best performance improvement is achieved at low SIR. For a SIR $=-5 \mathrm{~dB}$ and $L=4$ the BER is about $4 \cdot 10^{-4}$ for the metric in (9) against only $10^{-2}$ without shift. Moreover, when the signal to interference ratio increases, the MUD-MLSE receiver with $L=5$ converges towards the single-user detector without BER floor.

\section{CONCLusions}

The aim of this paper has been to demonstrate the performance of a reduced-complexity MLSE detector in the context of heterogeneous WLAN-like channels. The asynchronous behavior is due to the independent instantaneous channel impulse response realizations of the desired and interfering users, 


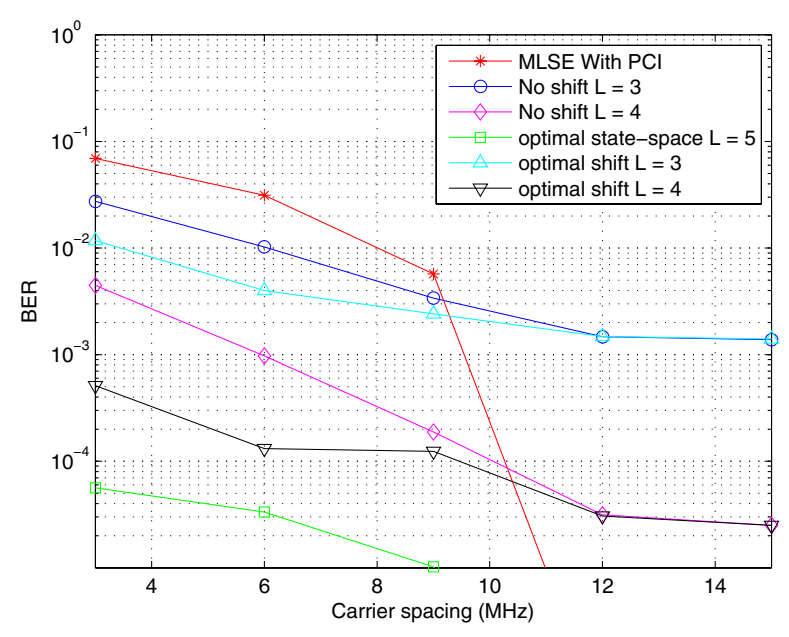

Fig. 4. Average BER versus channel overlap, labelled on Viterbi statespace $L$, with and without complexity reduction; $E b / N o=15 \mathrm{~dB}$, interference power $=$ signal power.

where the strongest paths of the interferer is time-shifted compared to the strongest path of the desired user. The interference cross-correlation terms hence yield their maximum at a nonzero time-lag. Based on this observation, we have proposed to shift the MLSE branch metrics so as to compensate for this asynchronism. This facilitates the construction of MLSE path metrics of reduced state-space, thereby significantly reducing the complexity of the detector. The simulation results point out how the shifted metric enhances the efficiency of a suboptimal MUD-MLSE detector.

Note that the high BER observed herein does not reflect the BER of a $802.11 \mathrm{~b}$ based WLAN system because neither DSSS neither CCK were considered. As future work, this technique will be introduced in a complete $802.11 \mathrm{~b}$ receiver. We expect, however, that the qualitative behavior will be the same as the one exposed herein.

The proposed MUD-MLSE detector of reduced complexity can be applied to other systems, which suffer a partial spectral overlap and asynchronous interference due to a strong frequency selective channel. We expect that with the marriage of emerging cognitive radios [18] and forthcoming spectrum liberalization [19], interference cancelation methods will play a vital role for future cellular, WLAN and ad hoc systems.

\section{REFERENCES}

[1] R. Diestel, Graph Theory, Springer-Verlag, 2000.

[2] S. Verdu, Optimum Multiuser Signal Detection, Ph.D diss., Univ. of IL., Urbana-Champaign,Aug. 1984.

[3] S.Moshavi, "Multi-user detection for DS-CDMA communications, IEEE Commun. Mag., vol. 34, pp. 124-136, Oct. 1996.

[4] Laster J.D, Reed J.H, "Interference Rejection in Digital Wireless Communications", IEEE Signal Processing Magazine, vol. 14, Issue: 3, May 1997 pp. 37-62.

[5] Andrews J.G, "Interference Cancellation for Cellular Systems: A Contemporary Overview", IEEE Wireless Communications Magazine, vol. 12, Issue: 2, April 2005 pp 19-29.

[6] H. Zamiri-Jafarian, S. Pasupathy, "Complexity Reduction of the MLSD/MLSDE Receiver Using the Adaptive State Allocation Algorithm," IEEE Transactions on Wireless Communications, vol. 1, no 1, January 2002.

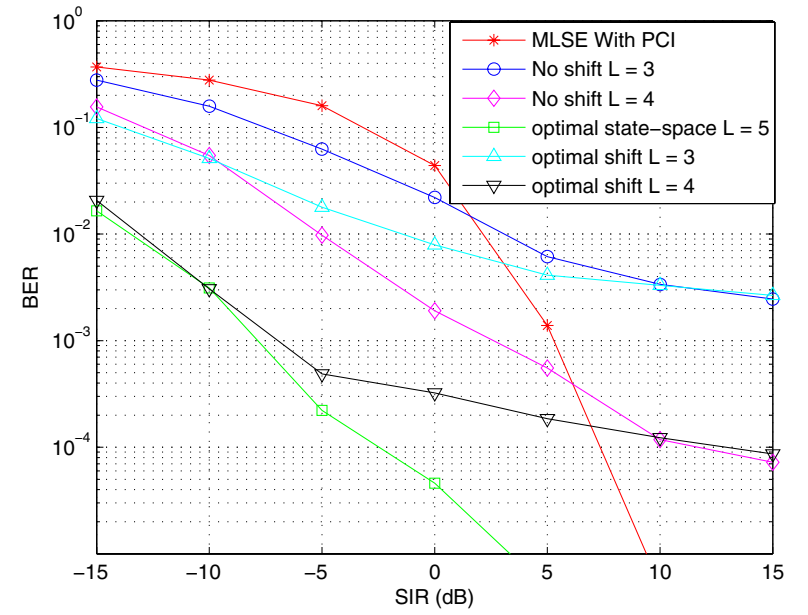

Fig. 5. Average BER versus interfering power, labelled on Viterbi state-space $L$, with and without complexity reduction; $E b / N o=15 \mathrm{~dB}$, channel overlap $=65 \%$.

[7] Z. Qin, et al., "Iterative Reduced-State Multiuser Detection for Asynchronous Coded CDMA," IEEE Transactions on Communications, vol. 50, no: 12, December 2002.

[8] P.B. Rapajic, B. Vucetic, "Adaptive receiver structures for asynchronous CDMA systems," IEEE J. Select. Areas Commun., vol. 12, no. 4, pp. 685-697, May 1994.

[9] I. Oppermann, "Extending the scope of 802.11 WLAN through LMMSE CDMA receiver structures," IEEE PIMRC 2002, Volume 2, 15-18 Sept. 2002, pp. 864-868.

[10] A.M. Kuzminskiy, Y.I. Abramovich, "Adaptive second-order asynchronous CCI cancellation: Maximum likelihood benchmark for regularized semi-blind technique," in Proc. ICASSP, vol. IV, pp. 453-456, May 2004.

[11] A.M. Kuzminskiy, C.B. Papadias, "Re-configurable semi-blind cancellation of asynchronous interference with an antenna array," in Proc. ICASSP, vol. IV, pp. 696-699, Apr. 2003.

[12] L.J. Pesik, M.A. Beach, D.P. McNamara, P.N. Fletcher, "Performance analysis of smart antenna systems for indoor wireless LANs," Third International Conference on $3 G$ Mobile Communication Technologies 2002, Conf. Publ. No. 489, 8-10 May 2002, pp. 418-422.

[13] T.W. Nuteson, G.S. Mitchell, J.S. Clark, D.S. Haque, "Smart antenna systems for wireless applications," IEEE International Symposium on Antennas and Propagation 2004, Volume 3, 20-25 June 2004, pp. 2804 - 2807.

[14] M. Ahn, D. Kim, J.S. Kenney, "Throughput improvement in interference limited multipath environments using a smart antenna for IEEE $802.11 \mathrm{~b}$ WLAN," IEEE Radio and Wireless Conference 2004, 19-22 Sept. 2004, pp. 411-414.

[15] S. Suthaharan, A. Nallanathan, B. Kannan, "Joint interference cancellation and decoding scheme for next generation wireless LAN systems," IEEE SPAWC 2003, 15-18 June 2003, pp. 284-288.

[16] B. Xu, C. Yang, S. Mao, "Multiuser space-time code for OFDM/SDMA systems [WLAN applications]," VTC 2004-Spring, Volume 2, 17-19 May 2004, pp. 828-832.

[17] H. Arslan, S. C. Gupta, G. E. Bottomley, S. Chennakeshu, "New Approaches to Adjacent Channel Interference Suppression in FDMA/TDMA Mobile Radio Systems", IEEE Transactions on Vehicular Technology, Vol. 49, No. 4, July 2000, pp. 1126-1139.

[18] Joseph Mitola III, Cognitive Radio: An Integrated Agent Architecture for Software Defined Radio, Ph.D diss., Royal Institute of Technology (KTH) Stockholm, Sweden, 8 May, 2000.

[19] M. Cave, "Independent Audit of Spectrum Holdings - Cave Report," Her Majestys Treasury, Ofcom, Dec. 2005. 